\title{
Simulating the Daylight Performance of Complex Fenestration Systems Using Bidirectional Scattering Distribution Functions within Radiance
}

\author{
Gregory Ward \\ Anyhere Software \\ Richard Mistrick \\ Penn State University \\ Eleanor Lee, Andrew McNeil, Jacob Jonsson \\ Lawrence Berkeley National Laboratory
}

January 2011 


\section{DISCLAIMER}

This document was prepared as an account of work sponsored by the United States Government. While this document is believed to contain correct information, neither the United States Government nor any agency thereof, nor The Regents of the University of California, nor any of their employees, makes any warranty, express or implied, or assumes any legal responsibility for the accuracy, completeness, or usefulness of any information, apparatus, product, or process disclosed, or represents that its use would not infringe privately owned rights. Reference herein to any specific commercial product, process, or service by its trade name, trademark, manufacturer, or otherwise, does not necessarily constitute or imply its endorsement, recommendation, or favoring by the United States Government or any agency thereof, or The Regents of the University of California. The views and opinions of authors expressed herein do not necessarily state or reflect those of the United States Government or any agency thereof or The Regents of the University of California. 


\title{
Simulating the Daylight Performance of Complex Fenestration Systems Using Bidirectional Scattering Distribution Functions within Radiance
}

\author{
G. Ward \\ Anyhere Software, 950 Creston Road, Berkeley, CA, 94708 USA
}

R. Mistrick, Ph.D.

Penn State University, 104 Engineering A, University Park, PA 16802 USA

E. S. Lee, A. McNeil, J. Jonsson, Ph.D.

Building Technologies Program, Environmental Energy Technologies Division, Lawrence Berkeley National Laboratory, Mailstop 90-3111, 1 Cyclotron Road, Berkeley, CA 94720 USA

\begin{abstract}
We describe two methods which rely on bidirectional scattering distribution functions (BSDFs) to model the daylighting performance of complex fenestration systems (CFS), enabling greater flexibility and accuracy in evaluating arbitrary assemblies of glazing, shading, and other optically-complex coplanar window systems. Two tools within Radiance enable a) efficient annual performance evaluations of CFS, and b) accurate renderings of CFS despite the loss of spatial resolution associated with low-resolution BSDF datasets for inhomogeneous systems. Validation, accuracy, and limitations of the methods are discussed.
\end{abstract}

Keywords: complex fenestration systems (CFS), bidirectional scattering distribution function (BSDF), Radiance software, windows, daylighting systems, shading systems, energy.

\section{Introduction}

As energy efficiency and indoor environmental quality objectives become increasingly aggressive in this charged environment to mitigate climate change, it becomes more important that window performance be quantified accurately. A routine, efficient method is urgently needed to model complex fenestration systems such as sunlight-redirecting mirrored louvers, holographic optical elements, and even conventional roller shades.

To address this problem, a method was defined to quantify solar gains through windows with nonspecular shading devices, termed complex fenestration systems (CFS), that bridged the gap between expensive solar calorimeter measurements and first-principles analytical models [Klems 1994a]. The method requires bidirectional optical measurements of non-specular layers such as shades or blinds, use of optical data for specular glass layers, and matrix layer calculations to combine these individual layer properties to produce overall window system transmittances and layer absorptances from which solar gains can be computed as a function of incident direction. This method is also applicable to the assessment of daylighting. 
Bidirectional transmittance and reflectance or scattering function (BSDF) data for nonspecular devices is critical for routine implementation of this method and obtaining such data has been and continues to be a non-trivial task. A detailed review of the methods used to characterize nonspecular materials is given by Andersen and deBoer [2006]: starting with the first scanning goniophotometer built in 1988 and validation of the calculation method [Klems et al. 1997] to today's heterogeneous methods encompassing measurement and simulation techniques [Jonsson et al. 2009; Andersen et al. 2009]. Modifications to the Window software were made to enable combination of layer data into any arbitrarily configured system of layers [Mitchell et al. 2008]; its database now contains BSDF data for some common shading systems. Other databases are also publicly available [DeBoer 2006], but differ in format.

In parallel, BSDF-enabled simulation tools have been and are currently under development. The DELight subroutine was implemented within EnergyPlus to enable daylight modeling of CFS using radiosity methods [Carroll and Hitchcock 2005]. Further modifications to EnergyPlus are underway to enable calculation of solar gains. Kaemph [2004] adapted the prism2 function within Radiance to model CFS, deriving two principle emerging directions from measured BTDF data. DeBoer [2006] implemented a method within Adeline which treats the CFS as a light-emitting surface.

This paper describes modifications made to Radiance [Ward 1998] which enable both visualizations and annual daylighting evaluations of CFS. A brief overview of the fundamental underlying method is given, then the methods of implementation within Radiance are described. Validation and limitations of the methods are discussed.

\section{Background}

Klems showed that with a few simplifying assumptions, it is possible to derive the overall system transmittance matrix of a set of layers, including all the effects of multiple interreflections between layers, from the optical properties of individual layers [Klems 1994a, 1994b]. Two approximations were made:

1. Optical properties are spatially averaged over a suitably-sized area so that spatially inhomogeneous systems, such as venetian blinds with repetitions of unit slats, are treated as homogeneous layers. Direct radiation through the blind would be a uniformly lit patch rather than a series of stripes, which was deemed sufficient for the purpose of solar gain calculation. Spatial averaging for two parallel layers with spatial inhomogeneities of comparable size will not be correctly treated: e.g., two parallel, partially-closed blinds positioned such that the opening between slats on one blind is aligned with the closed slat of the second blind will not result in zero direct transmittance.

2. The original method described in 1994 assumed properties that were measured using a source that very roughly approximated the solar spectrum after transmittance through a layer of glass; i.e., spectrally averaged and unpolarized. However, Window 6 implemented the Klems method and applies the matrix layer calculation method at each wavelength, if detailed spectral information are available for the individual layers, to produce spectral-angular system properties which can then be used directly or appropriately averaged. Interference and polarization effects are at present not accounted for.

With these approximations, the solar optical properties of a system can be defined in terms of its bidirectional transmittance and reflectance distribution functions [Nicodemus 1965], which are in effect a set of hemispherical luminous coefficients defined by paired incident and outgoing angles. The incoming and outgoing hemispheres are subdivided into a grid of elements (referred to as a "basis") and the radiance is averaged over each solid angle element or "patch". To obtain total transmitted radiation for a given incident angle, $\theta_{1}, \varphi_{1}$, the luminous coefficients are multiplied by the incident irradiance and summed for all patches of the hemispherical basis using a matrix calculation, as in (1), 


$$
\tau\left(\theta_{1}, \phi_{1}\right)=\int_{0}^{2 \pi} \int_{0}^{\pi} B \operatorname{RDF}\left(\theta_{1}, \phi_{1}, \theta_{2}, \phi_{2}\right) \cos \theta_{2} \sin \theta_{2} d \theta_{2} d \phi_{2}
$$

or,

$$
\tau\left(\theta_{1}, \phi_{1}\right)=\sum_{k=1}^{145} B \operatorname{DDF}\left(\text { patch }_{k}\right) \int_{0}^{2 \pi} d \phi_{2} \int_{0}^{\pi} \cos \theta_{2} \sin \theta_{2} d \theta_{2}
$$

where, $\theta$ and $\varphi$ (Figure 1) define the boundaries of each patch of the basis and (2) shows integration of flux over the Klems full 145x145 basis, which is the default basis used in Window $6 .{ }^{1}$

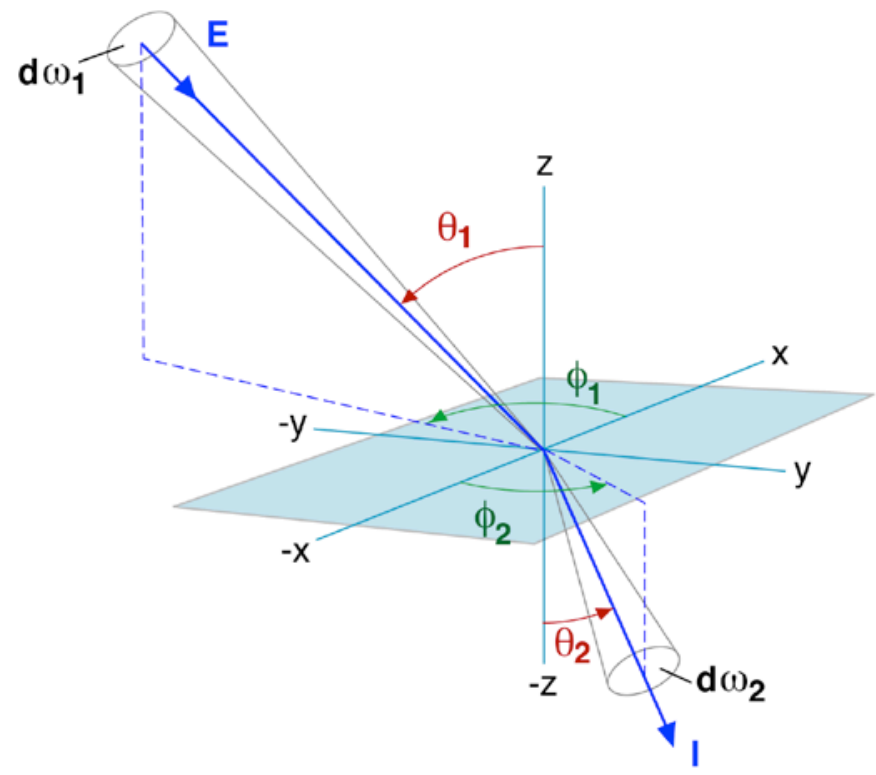

Fig. 1. Coordinate system for bidirectional measurements

For multi-layer calculations, output flux can be used as input for the next layer, enabling one to create a coplanar window system from an arbitrary configuration of individual layers (e.g., glass layer, between pane shade, glass). The method assumes that the layer planes are infinitely long compared to the depth of the system $(\mathrm{l} / \mathrm{d}>5)$, ignoring the edge effects of the window frame. Similarly, total reflected radiation for a multilayer system can be computed. The outgoing total radiation is then distributed to interior room surfaces to calculate solar gains.

When this method is applied to daylighting assessments, spatial averaging over a patch results is perhaps the most limiting aspect of this method. The spatial resolution of outgoing flux is as coarse as the coordinate system or basis and hence, one cannot resolve features smaller than that. Radiance provides work-arounds for some of these issues, which is discussed in the following sections.

\section{CFS modeling in Radiance}

\footnotetext{
${ }^{1}$ Klems modified the Tregenza hemispherical subdivision to give higher resolution in incident angle and a weighting of the patches proportional to their solid angle and projected area. The angular resolution of this basis is approximately $\pm 5^{\circ}$ in incident angle and much coarser in azimuth.
} 
Until recently, there was no way to effectively utilize general BSDF data in Radiance. Such data were difficult to obtain, and representation standards were lacking. With the development of Window 6, these barriers are lifting.

The backwards ray-tracing approach in Radiance needs to know a priori where to look for light sources and where to find peaks in the BSDF, so we know where to send our samples for optimal results. The standard approach for CFS in Radiance is to precompute the light passing through each window or skylight, treating it as a "white box" in the final rendering, otherwise known as a "secondary light source." The original mkillum program was written to convert a CFS, described as geometry and materials, into a secondary light source with a candlepower distribution corresponding to a particular exterior condition (i.e., sun, sky, obstructions, reflections, etc.). In this mode, mkillum is computing all the light interactions of whatever CFS might be placed in the opening. During rendering, thus-converted windows and skylights will usually have a dual nature, applying the mkillum-computed light distribution for illuminating interior surfaces, while applying the original CFS geometry and materials for direct viewing and beam penetration. Computing light at a point on an interior surface then involves tracing "shadow rays" to all light sources, including secondary sources that turn into their original geometry when intersected. In the case of venetian blinds, this process provides a representative view out the window, since the actual blinds geometry is represented, plus striped shadows on the floor when sunlight penetrates.

While the standard mkillum technique just described works well most of the time, there are important cases where it fails. Using a backwards ray-tracing approach, it is nearly impossible to account for sunlight transmitting through a system with curved, specular elements. However, if we are given the BSDF as input, we can apply it directly to convert the exterior illumination arriving at the window or skylight into an interior candlepower distribution, as we describe in the following section. A photon-mapping extension (i.e., forward ray-tracing) may also be used for such systems, although it fundamentally changes the way Radiance works [Shregle \& Weinhold 2004]. Neither approach is practical for annual simulation, since a separate precomputation step is required for every solar condition. A daylight coefficient (DC) method is needed.

The Daysim version of Radiance was developed specifically for annual simulation using daylight coefficients [Reinhart \& Herkel 2000], but it cannot take advantage of mkillum in its calculations. All light passing into the space must be accounted by the single-step backwards ray-tracing approach in Radiance, making venetian blinds and other CFS a serious challenge. Particularly if the CFS is operable, the basic DC approach can become quite expensive. In Section 3.2, we describe a new DC method that separates the effect of the BSDF, making annual simulations truly practical, even with complex, operable fenestration.

In the following sections, we describe two techniques for incorporating BSDF data in Radiance. The first technique enhances mkillum so it can precompute light output from CFS portals (windows or skylights) using BSDF data, permitting a broader array of systems to be modeled. The second technique extends the DC approach using the rtcontrib program to compute coefficients for incoming and outgoing directions on CFS with BSDF data connecting the two. This offers advantages for annual simulation and operable shading devices, as changing the control setting is as simple as swapping matrices in an inner time-step loop.

\subsection{Single time-step evaluations and renderings of CFS in Radiance using mkillum}

The main challenge with CFS is that their output is a) difficult to compute, and b) varies over their surface. While it is possible to rely upon the default Radiance interreflection calculation to integrate light arriving through the windows and skylights, the more complex their behavior, the more time it takes to get a reasonable result. Since we know that windows are important contributors to illumination, we can turn them into secondary light sources, precomputing and smoothing their output distributions for efficient rendering. This is the purpose of mkillum.

Since Radiance 3.9 was released in 2008, mkillum has supported BSDF descriptions of window and skylights, using XML files exported from Window 6. A key advantage is that mkillum does not need to model how light gets through the window, since the BSDF informs us directly. All mkillum needs to determine is how light is impinging on the exterior opening. 
The obvious way to compute the external incident radiation is to send sample rays out from the window into the exterior environment. Obstructions, interreflections, overhangs, buildings and all will be accounted for by this external sampling. However, we need to make special allowances for light sources such as the sun, which constitute important, localized contributors to illumination. Random sampling is no way to integrate the sun, even if we can account for any complex redirection to the interior with a BSDF. We therefore send a user-selected number of samples from random points on each opening's exterior towards the sun and any other localized sources described in the scene model. The radiation from these samples will be accounted for separately and passed to the appropriate incoming direction of the BSDF. ${ }^{2}$ What happens in the final stage depends on the setting of the mkillum 'l' flag.

The 'l' flag indicates whether the window being computed is a secondary source ('l-') or a standard light source (' $1+$ '). In the latter case, all contributions are fed through the BSDF and resampled to create a candlepower distribution in a mkillum-specific polar coordinate system for treating the window as if it were a luminaire. This is not normally done for windows, since a direct view will show only a uniform output corresponding to the average behavior in the viewer's direction. Also, for any window system with an unobstructed beam component, the resolution of the BSDF and the recorded candlepower distribution will tend to smear the light path excessively, resulting in blurry and featureless shadows. This is why the default method computes a secondary light source ('l-'), leaving the final rendering phase to create a realistic view out the window. Similarly, direct contributions passing through the window are also rendered, such as the detailed shadows cast by venetian blinds, which would be nearly impossible to produce using a BSDF [de Boer 2006].

In the secondary source ('l-') method just described, a direct beam through a window is accounted in the final rendering phase, so we must discount this contribution in the candlepower table. To accomplish this, we make a separate tally of all source contributions when accumulating our incoming radiation. In the final mkillum stage, as we pass this light through our BSDF and redistribute the output into a candlepower table, we subtract source light that passes straight through the BSDF and continues in the same direction. Light that is redirected by the BSDF is unchanged. The user then creates a geometric representation of the CFS for proper view and beam shading. The rendered result is an accurate depiction of the window geometry, the indirect contribution from the window, and the direct contribution passing through the window (Figure 2).

While the calculation of luminance distributions for CFS characterized with BSDF data is fairly efficient using mkillum, it still takes some time to sample the exterior environment, account for interreflections and so on, then considerably longer to render a final image using the computed secondary light sources. The time is well-spent if the desired output is a high-quality rendering at a particular time and day, but may be impractical in an annual simulation context. A DC approach is required.

\footnotetext{
${ }^{2}$ If a source is large or local, its energy may enter multiple patches in the BSDF basis. If one of our previous random samples happens to hit a light source, it is given zero energy to avoid double-counting.
} 

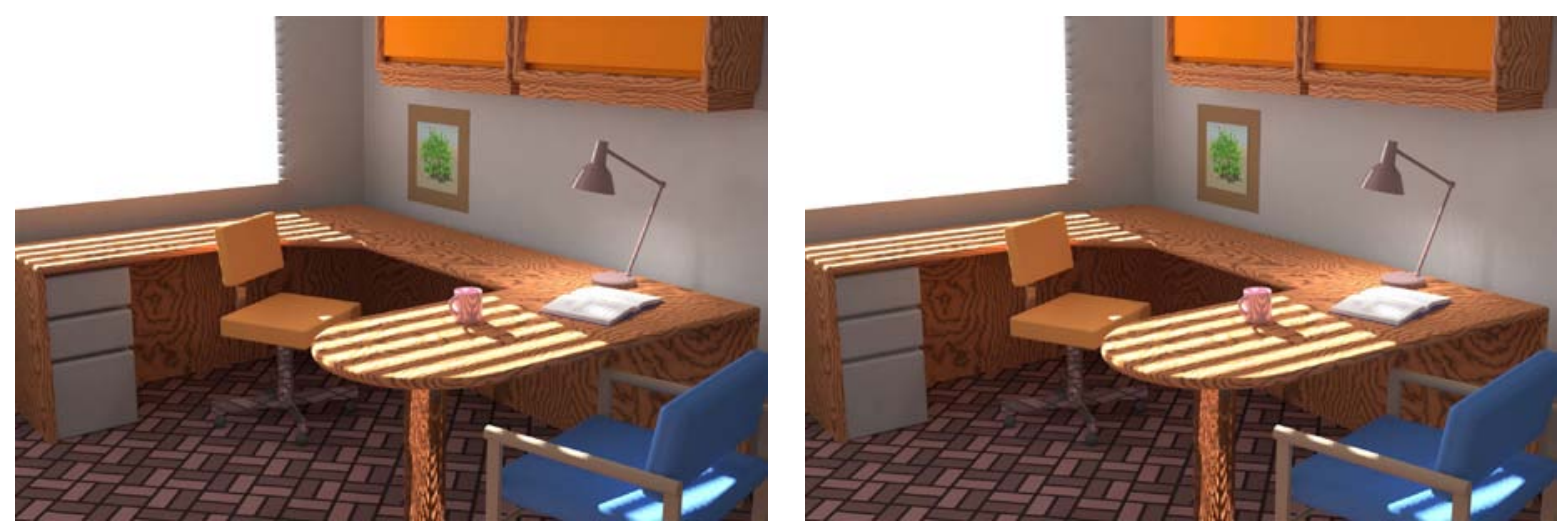

a

$\mathrm{b}$
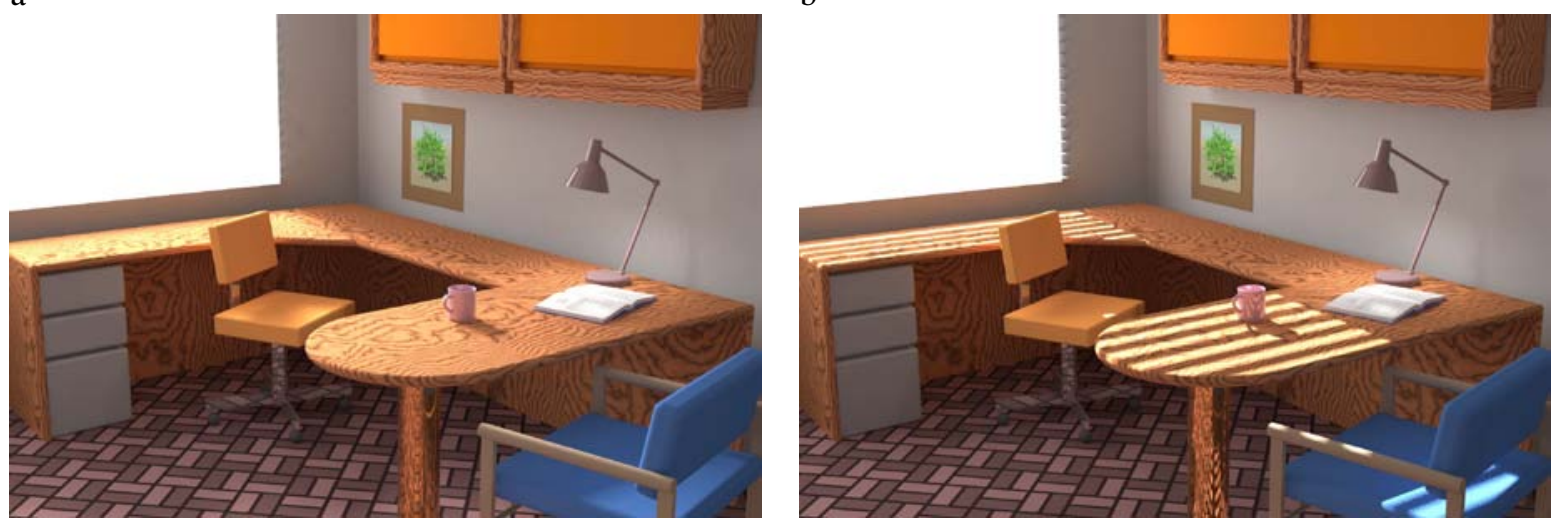

d

Fig. 2. Office with Venetian blinds showing a) standard backwards ray-traced rendering without mkillum or BSDF data (some sampling artifacts are visible on the wall near the desk), b) mkillum l- without BSDF data (note smoother shading on wall near desk), c) mkillum I+ where window is a standard light source with a computed candlepower distribution (the average behavior is correct but appearance of blind and shadows are lost), and d) mkillum l- with BSDF data and blind geometry allows direct beam penetration with shadows from the blind slats.

\subsection{Annual time-step calculations of CFS in Radiance using rtcontrib}

The DC approach is a proven method for annual simulation that subdivides the sky into patches whose partial contributions are computed independently [Tregenza 1983]. Any general lighting simulation method may be used to compute the daylight coefficients in advance of the annual loop. The inner timestep then assigns a set of values to the sky patches for a particular day/time/weather condition, multiplying this against the precomputed daylight coefficients and summing the partial contributions. This vector dot product is quick to evaluate, making the DC precomputation well worth the effort, but the standard approach must be altered substantially to handle BSDF data and operable systems.

We have the same issues in a DC calculation as we do in a single time-step rendering, which is the complex interaction at the windows. Unfortunately, mkillum cannot help us here, because it only computes the output distribution for a particular daylight condition. We want to know what happens under any condition, which would require a separate run of mkillum for every sky patch. While certainly possible, such a calculation is very inefficient and would take too long to complete. This is where we introduce the rtcontrib tool.

The rtcontrib tool can compute light flux transfer between almost arbitrary sources and destinations using a general geometric ray-tracing method. In the context of daylight coefficients and CFS, we use rtcontrib to calculate a matrix linking sky patches to incident directions on a particular window or skylight group. We call this matrix D for "daylight," and we compute separate D matrices for each fenestration group, where groups are divided by elevation (orientation) and proximity to external obstructions, an issue we explore more fully in Section 4. From matrix $\mathbf{D}$ and a set of sky patch luminances, we can compute the luminance arriving from specified directions on each window group, as all daylight illumination ultimately comes 
from the sky. ${ }^{3}$ This is illustrated in Figures 3b-3d, which show a Tregenza sky subdivision outside the window shaded pink in Figure 3a through a Klems basis. BSDF patches that see only sky will get a contribution from a few sky patches, whereas BSDF patches that see other surfaces in the scene will receive an integration of many sky patches. The $\mathbf{D}$ matrix thereby incorporates all daylight contributions, direct and indirect, accounting for diffuse and specular reflections, obstructions, etc. In addition, we provide a single ground patch for the distant horizon (shown in Figure 3d), since we cannot specify an infinite ground plane as Radiance geometry.
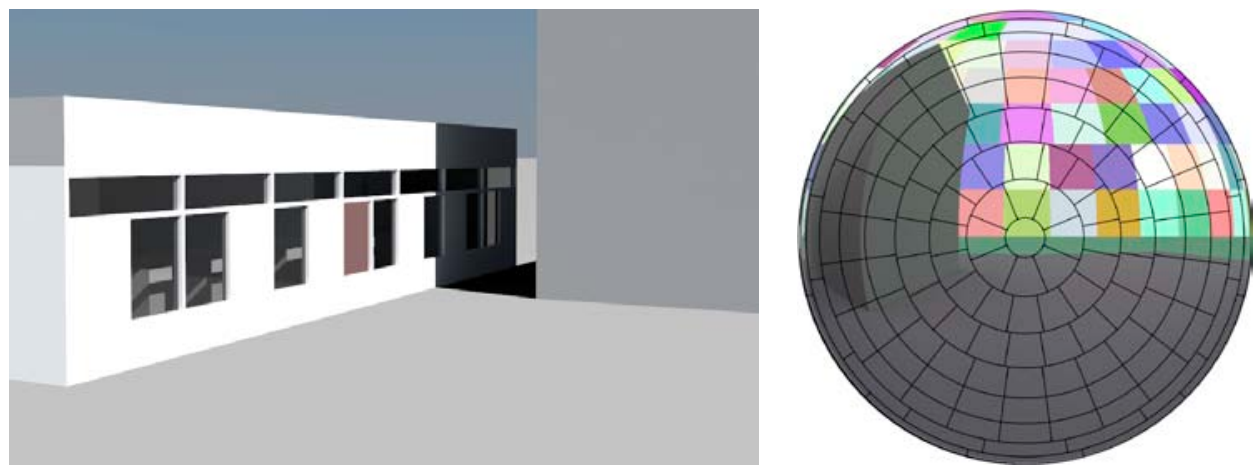

$\mathrm{b}$
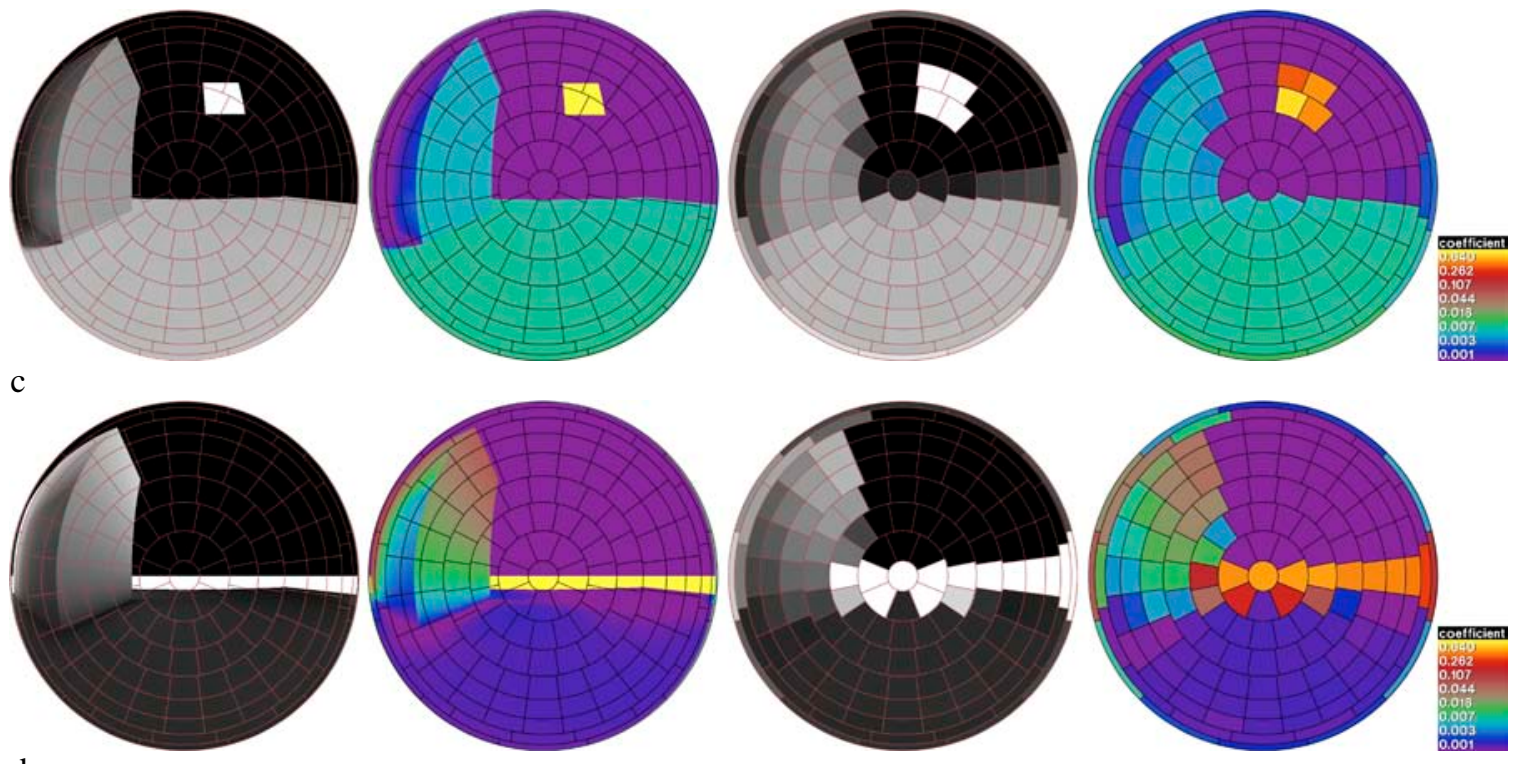

Fig. 3. a) Selected window (shaded pink) to illustrate incident daylight matrix $D$ and interior view matrix $V$, b) matrix $D$ relates sky patches to Klems BSDF patches, c) renderings of contributions from Tregenza patch 74 (left two images) and visualizations of the D matrix coefficients for the same Tregenza patch (right two images) - reflections from model geometry (ground polygon and adjacent building) are included in the $D$ matrix, and d) renderings of contributions from the ground patch (left two images) and visualizations of the $D$ matrix coefficients for the ground patch (right two images). Because this model includes a ground polygon, the ground patch fills the space between the edge of the ground polygon and the horizon.

For a given sky condition, we could quickly compute the outgoing luminance on the window interior from a BSDF matrix. We could use these candlepower distributions to render an image directly, but as this is also part of our time-step calculation, it would be much better to precompute this transfer function as well. A second run of rtcontrib is used to relate the exiting directions on our window interiors to the desired image or sensor values, and we call this the $\mathbf{V}$ or "view" matrix. This matrix is illustrated in the case of an interior view, shown as projected colors in Figure 4.

\footnotetext{
${ }^{3}$ For the time being, the sun is assumed to have its energy distributed to a few sky patches in our analysis.
} 


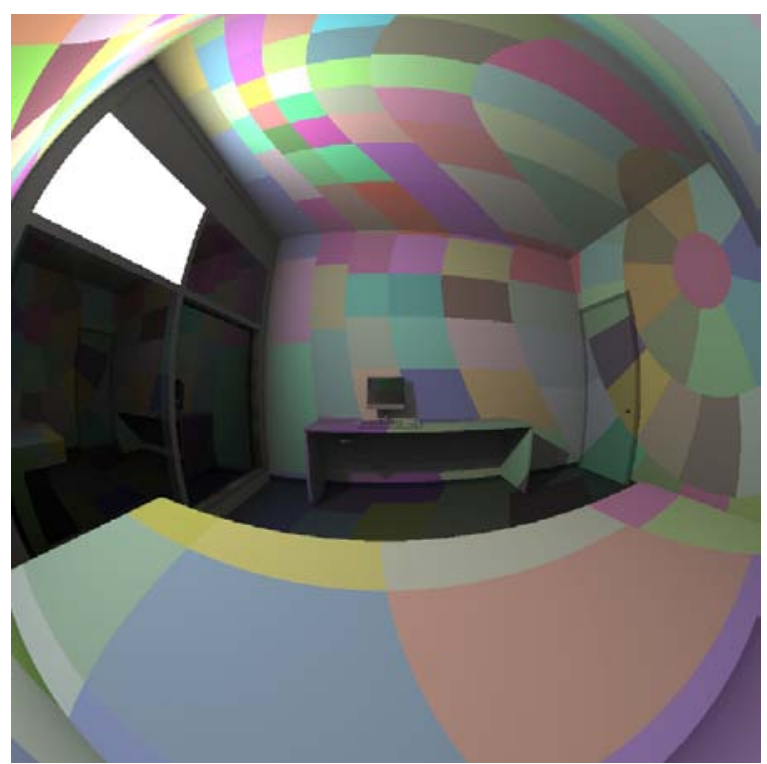

Fig. 4. A random-color illustration of the view coefficients $\mathbf{V}$ corresponding to the exiting directions of the Klems BSDF basis.

Identifying the BSDF matrix as $\mathbf{T}$, we can write our combined matrix formula for a single window group thus:

$\mathbf{i}=$ VTDs

where:

$\mathbf{i}=$ result vector (luminances, illuminances, image, etc.)

$\mathbf{V}=$ view matrix, relating outgoing directions on window to desired results at interior

$\mathbf{T}=$ transmission matrix, relating incident window directions to exiting directions (BSDF)

$\mathbf{D}=$ daylight matrix, relating sky patches to incident directions on window

$\mathbf{s}=$ sky vector, assigning luminance values to patches representing sky directions.

The dimensions of the vectors and matrices will depend on the desired resolution in the calculation. If we are computing 100 interior illuminances from a Tregenza sky subdivision with 146 patches (including ground), then the $\mathbf{s}$ vector will be $146 \times 1$, the $\mathbf{D}$ matrix will be $145 \times 146$, the $\mathbf{T}$ matrix using a full Klems BSDF basis will be 145x145, the $\mathbf{V}$ matrix will be 100x145, and the resultant $\mathbf{i}$ vector will be 100x1.

When multiple window groups are involved, we evaluate vector $\mathbf{i}$ for each group and sum the results together. For operable fenestration systems, the above formula permits us to quickly swap the $\mathbf{T}$ matrix for an alternate position (e.g., slat angle) and iterate until we arrive at the desired result vector $\mathbf{i}$. In cases where the CFS is not operable, we can collapse the $\mathbf{V}$, $\mathbf{T}$, and $\mathbf{D}$ matrices into a single rectangular daylight coefficient matrix for the sake of efficiency. The important point is that we have eliminated all ray-tracing calculations from the inner time-step loop, converting it into a straightforward matrix-vector multiplication. This technique has been used successfully in a pilot study of 60 real buildings [Saxena et al. 2010].

Figure 5 shows our window office again, this time rendered using rtcontrib. Precalculation of the $\mathbf{D}$ and $\mathbf{V}$ matrices took about an hour on a laptop computer, followed by 12 seconds to evaluate the $\mathbf{i}$ vector (1.5 megapixels long) for a particular date, time, and slat angle. Once again, we have lost information for the slat shadows, but in this case, we have no convenient remedy as we are relying completely on the BSDF to transfer external radiation to the interior. (The issue of BSDF resolution is addressed in the following section.) 


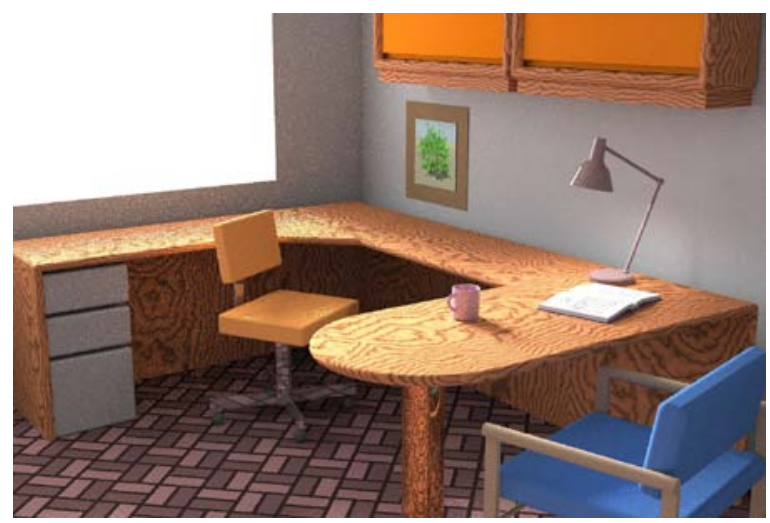

Fig. 5. This rendering was computed using rtcontrib and the matrix equation given in Section 3.2. The noise is an artifact of a more basic Monte Carlo path tracing method required by rtcontrib.

\section{Validation and applicability of simulation methods}

\subsection{Assignment of flux in exiting direction within mkillum and effect on renderings}

To ensure that the Window 6 BSDF data coordinate system was properly interpreted within Radiance, we ran a simple check to validate input and output directions by reproducing BSDF output using mkillum. A simple test of an incident normal light ray that redirects light into specific Klems patches verified that zone directions are being correctly addressed by mkillum (and therefore by rtcontrib).

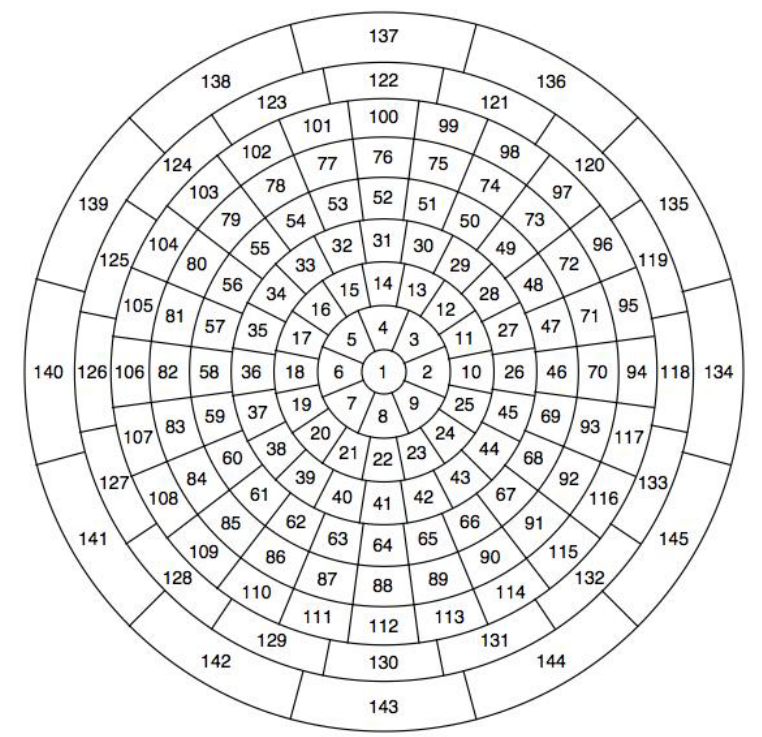

Fig. 6. Klems 145-patch hemispherical basis with numbered subdivisions

In evaluating the single patch output, which considered output in patches numbered 1, 5, 25, 30, 63, 72, 96, 107, and 138 (Figure 6), it is clear that light expected to be redirected into a single patch is distributed over multiple patches (Figure 7). This is due to the differences between the Klems basis and polar representation of the distribution that is created and applied by mkillum: incoming flux from a Klems patch must be split up and assigned to outgoing patches of the polar basis, which differ slightly in angular position. Neighboring patches which had BTDF values of zero are receiving some of the flux from adjacent patches that contained individual spikes in the distribution.

The ' $1+$ ' and ' $1-$ ' options also provide different results. As described above, with the mkillum ' $1+$ ' option, the specular component is determined from the BSDF data. Using the 'l-' option, the specular component is removed and is addressed using a description of the material's geometry which is placed within a zone of 
designated thickness adjacent to the planar BSDF material. Figure 7 shows the differences in performance for a small rectangular aperture facing the inside of a hemisphere. In this example, the eight patches with non-zero values cause some light to be distributed beyond their boundaries. The central patch of the polar representation is much larger than the Klems patch 1.

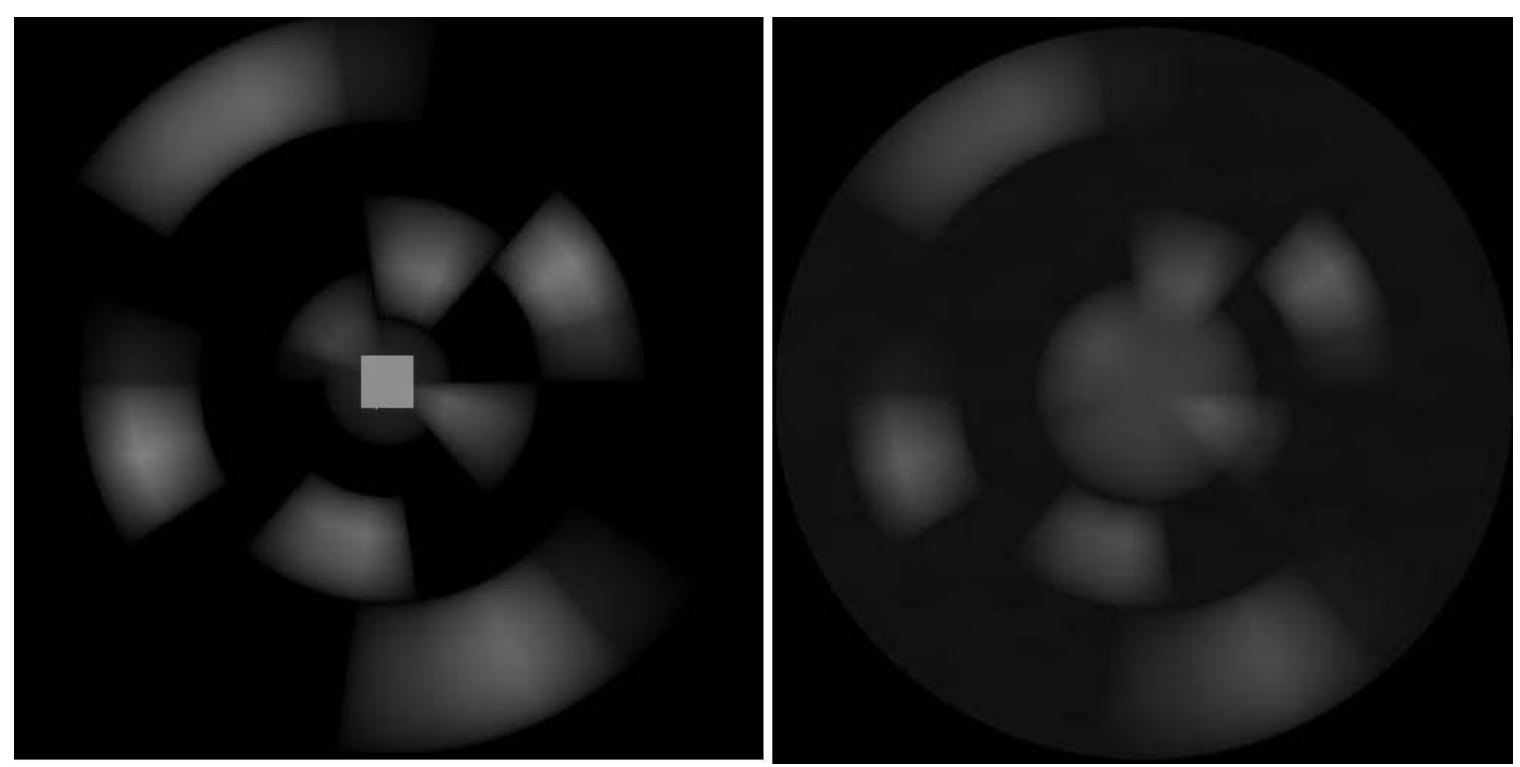

Fig. 7. Graph of direct illuminance provided on the inside of a hemisphere from a small rectangular BTDF material illuminated perpendicular to its surface. Nine of the 145 patches have non-zero BTDF values. Image at left applies $\mathbf{m k i l l u m}$ with the ' $\mathrm{l}$-' option. The center rectangle is the light that passes directly through the rectangular aperture. Image at right applies mkillum with the 'I+' option. Note how output in the center patch (1) extends into the neighboring patches (2-9). Similarly, the other eight patches extend beyond the extents of a single Klems patch.

To address performance with real materials, validation tests were conducted using mkillum with the ' $1+$ ' and 'l-' settings for a horizontal blind (calculated in Window 6) and a fabric shade (measured with a goniophotometer). Both shading devices permit direct sunlight to penetrate at the specular angle. The results of these tests show that mkillum is correctly able to track the amount of flux admitted to the space quite accurately under both the ' $1+$ ' ' and ' $l-$ ' approaches, but is unable to properly track the flux direction when there is a sharp spike in the distribution and the ' $1+$ ' option is applied. The flux that passes directly through the material and exits within one of the 145 patches is often distributed across multiple patches.

In the case of the horizontal blinds (l+), the flux in patch 4 is approximately $21 \%$ of its theoretical value, while in patch 1-3 and 5-9 it is roughly seven to twelve times the theoretical value determined using the BTDF, and in patches 13, 14 and 15, it is 37 to 87 times higher (Figure 8a). In all cases, the total flux emitted by the material over all directions is still very close to the theoretical value. When the direct flux is removed from the BSDF data using the 'l-' option, the total emitted flux and the directional information are quite accurate.

Examples of the theoretical and mkillum derived center zone candlepower and total flux for a horizontal blind and fabric shade material with open weave are shown in Figure 8 for the two different mkillum options. Separate tests at angles where a concentrated direct source beam is completely blocked by the shade, as well as a test where a uniformly luminous hemisphere illuminated the BTDF material, showed excellent agreement across all patches. Since the basis definition for incoming and exiting flux is the same in rtcontrib, the spread of flux to adjacent patches seen in mkillum is not a concern in rtcontrib.

The results of these tests show that BSDFs can be applied with mkillum, but beam contributions that pass directly through shading devices should be separated out from the BSDFs using the 'l-' option to permit this flux to be properly addressed. Very narrow spikes in the output distribution resulting from specular reflections within a CFS are unlikely to be treated accurately by either approach, since flux will be distributed across multiple patches as shown in Figure 7. 
a

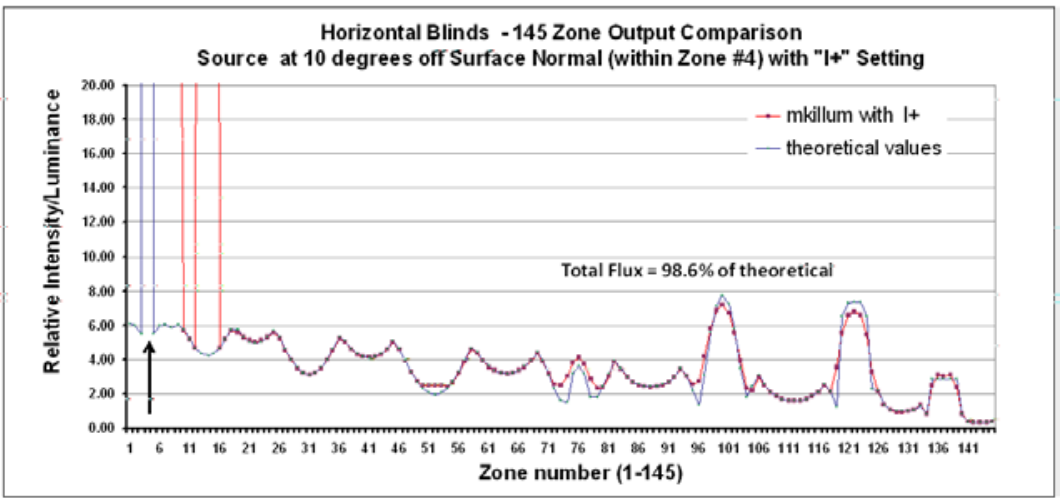

b

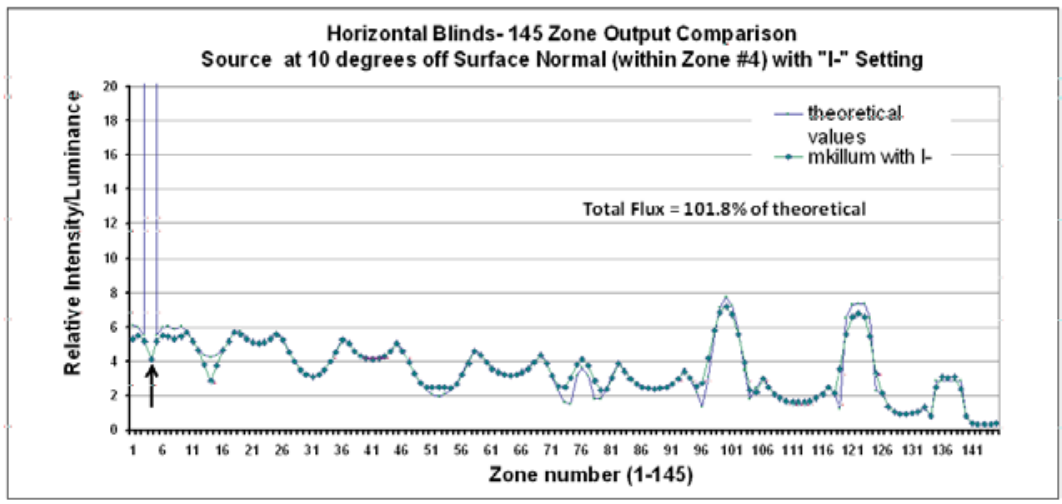

C

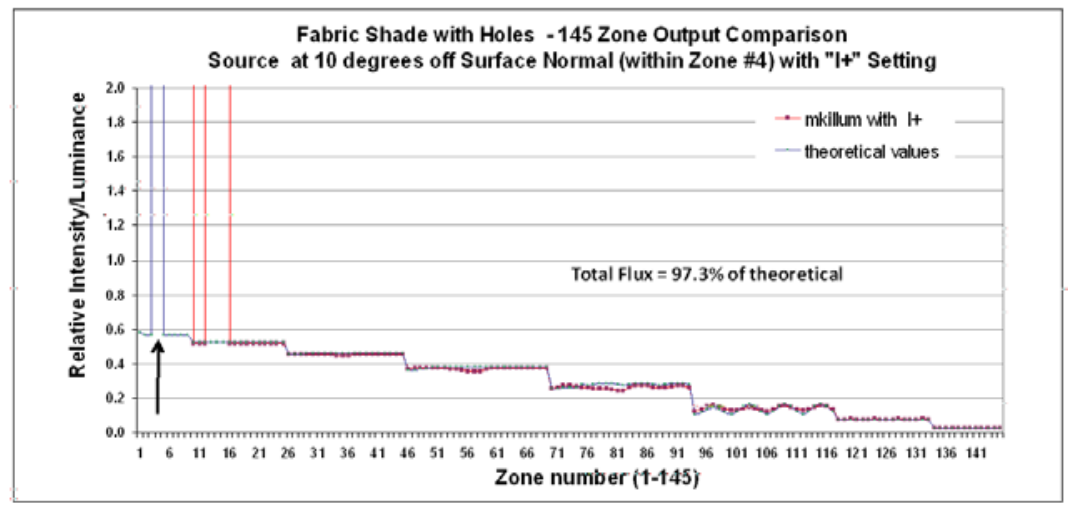

d

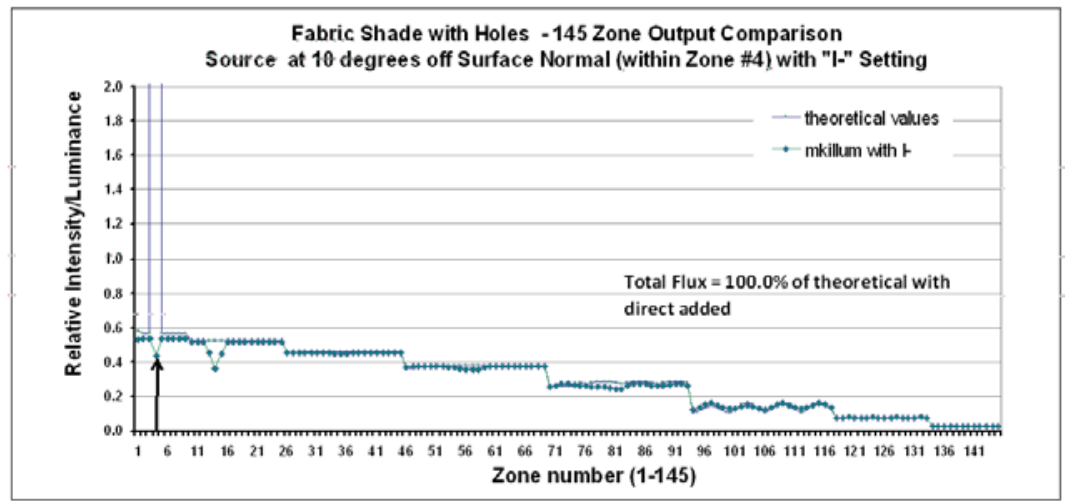

Fig. 8. Graph of $m$ killum's performance using both the ' $1+$ ' and ' 1 -' options for the illuminance at the center of each of the 145 patches of a Venetian blind and fabric shade when illuminated $10^{\circ}$ from the surface normal (within patch 4 at arrow), where direct light is permitted to pass through the material. The graph for 'I-' excludes the direct light, but it has been added for the total flux comparison. 


\subsection{Resolution of the BSDF and sky subdivision and effects on accuracy}

Up to now, we have discussed hemispherical subdivision schemes with 145 patches (full Klems BSDF basis and Tregenza for sky subdivisions), but because values are averaged over patches, the resolution of the hemispherical division schemes affects accuracy. A resolution of 145 patches equates to an average solid angle of 0.0433 steradians per division (cone with $13.5^{\circ}$ apex angle). For comparison, the sun has a $0.5^{\circ}$ apex angle.

For the sky subdivision, the Radiance program used to create the $\mathbf{s}$ vector spreads the radiance of the sun over three Tregenza sky divisions (a $0.5^{\circ}$ source spread over three $13.5^{\circ}$ patches) to maintain the appropriate centroid. In addition, the sky divisions do not align with Klems divisions causing the solar radiance to be spread over six or more Klems patches. If the simulated CFS has an output peak corresponding to sunlight transmission or re-direction than what may in reality be a focused patch of light may be spread up to $20^{\circ}$ off axis. To reduce this effect, a higher resolution subdivision of the sky such as the Reinhart scheme, can be used which further subdivides the Tregenza patches while preserving a zenith patch (Figure 9).

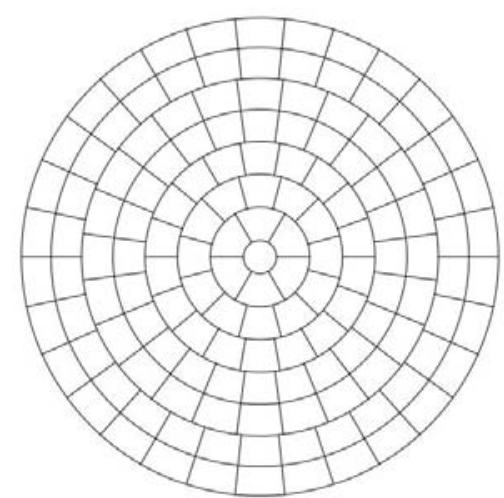

a

Fig. 9. Orthographic projections of sky division schemes (a) Tregenza - 145 divisions, (b) Reinhart MF:2 - 581 divisions, and (c) Reinhart MF:4 - 2321 divisions.

Incorrect flux placement can also occur when a low-resolution BSDF is used to represent specularlyreflecting CFS with peaky output. Averaging over the Klems 145-patch basis reduces the intensity and spreads the narrow peak over a larger angle. The redirected sunlight would cover a larger portion of the ceiling and be less bright at any single location.

To better understand impacts, high-resolution angle bases were created to better understand impacts on simulation accuracy (Figure 10). A variable resolution angle basis would most efficiently characterize a peaky optical redirection system. The division schemes depicted are fixed resolution schemes, but might represent the finest level of resolution for a variable division scheme. Images in Figure 11 illustrate the size of BSDF patches for three bases resolutions. 


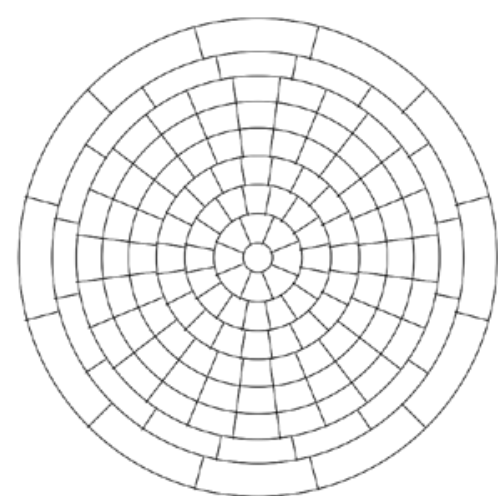

a

Fig. 10. Orthographic projections of BSDF hemispherical division schemes (a) full Klems basis - 145 patches, (b) 2x Klems580 patches, and (c) $4 x$ Klems- 2320 patches.
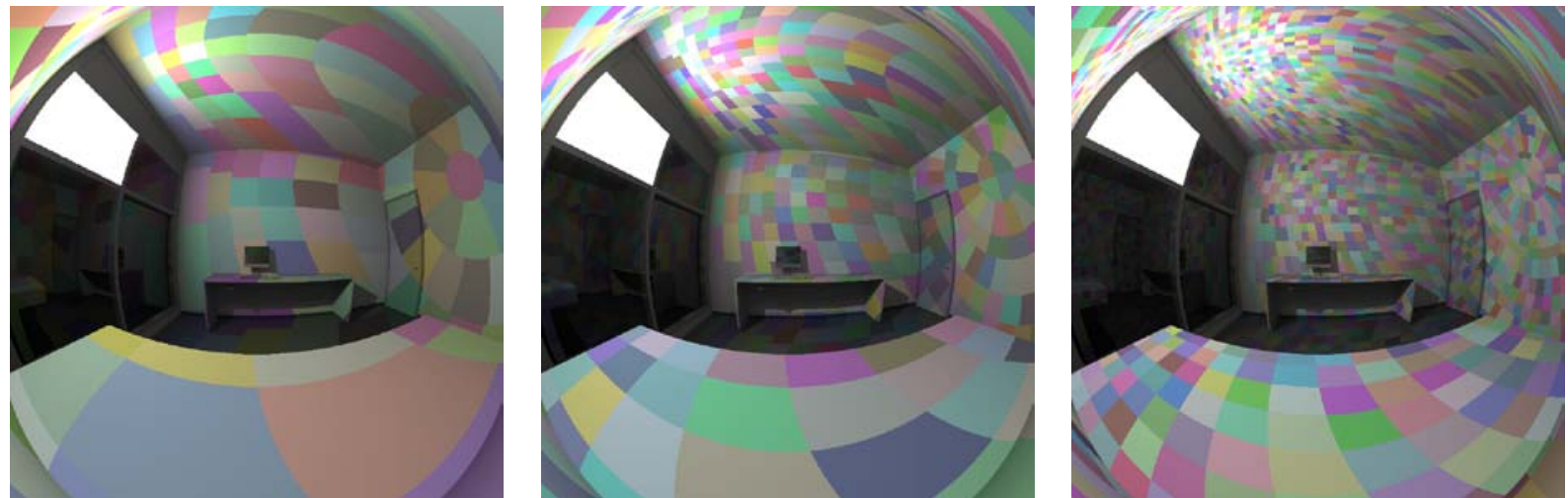

Fig. 11. Renderings illustrating size and locations of out going BSDF patches for (from left to right) full Klems basis, 2x Klems basis, and $4 x$ Klems basis.
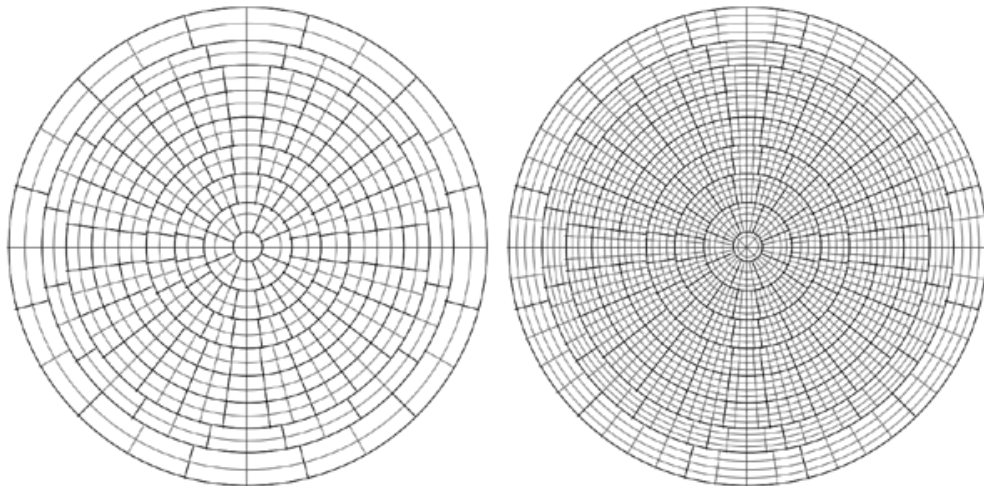

C 


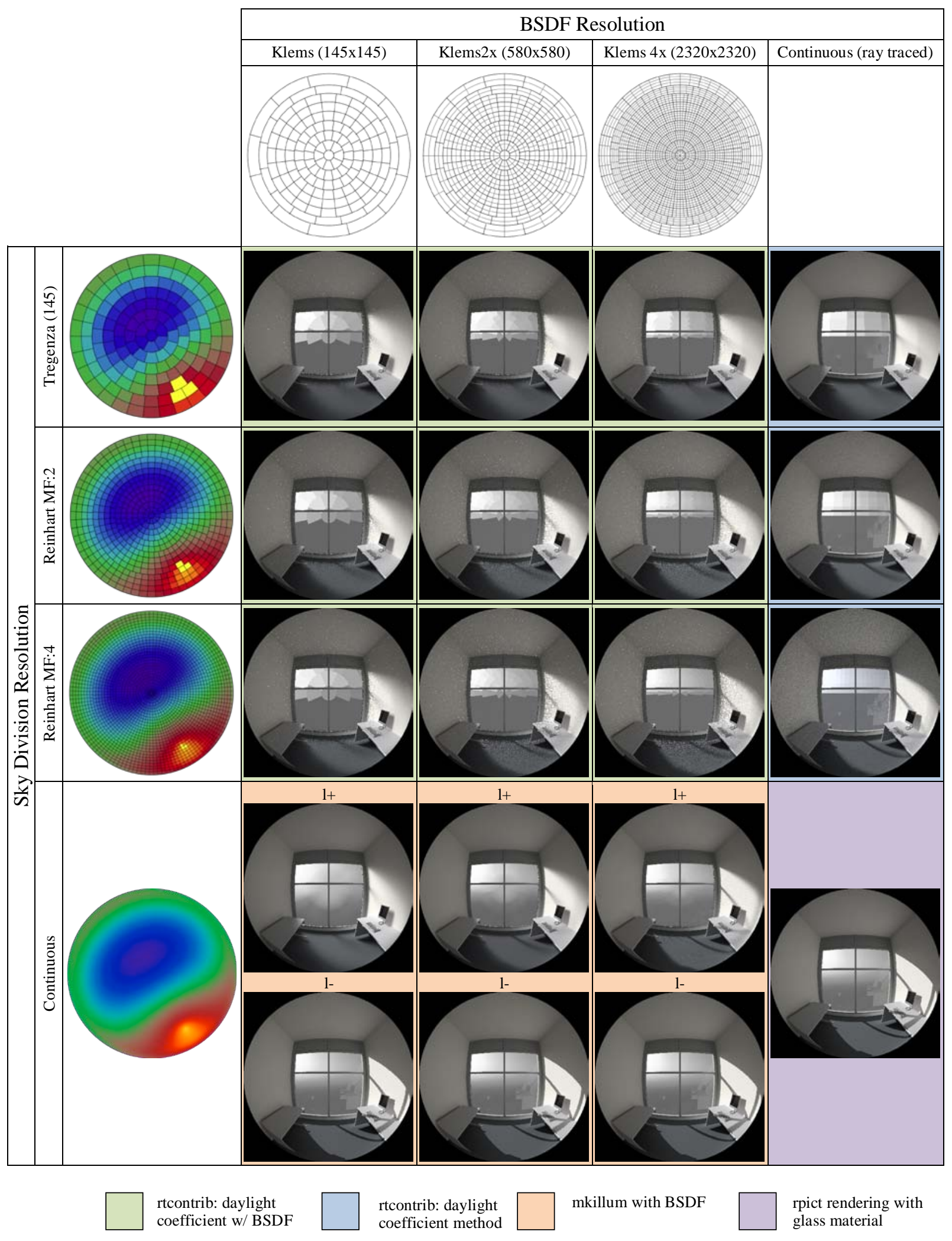

Fig. 12. Renderings using different BSDF and sky division resolutions. The color of the box indicates the method used to generate the image. 


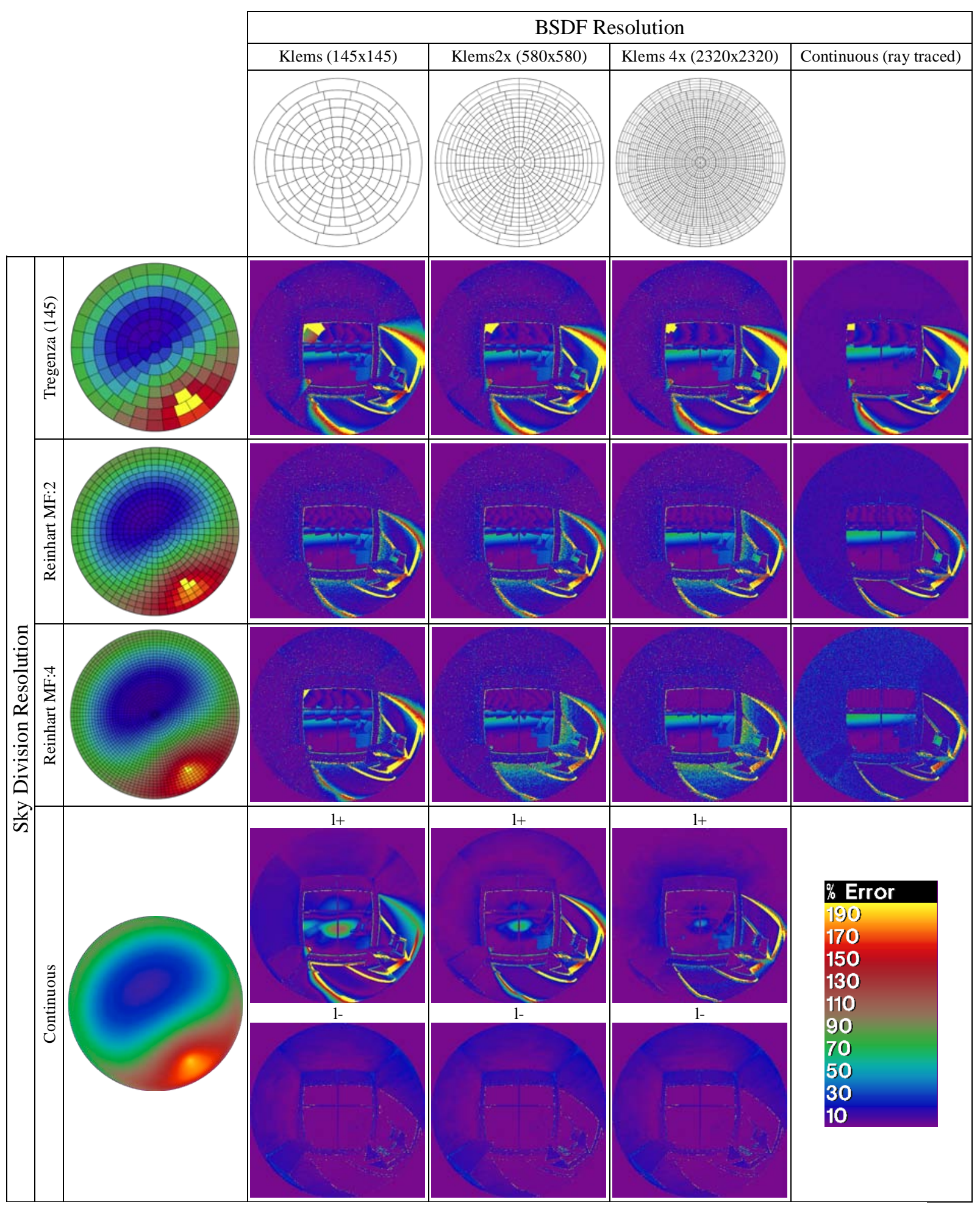

Fig. 13. Percent difference falsecolor renderings - images illustrate the percent difference between the image in the box with the fully ray-traced rendering.

Images in Figure 12 demonstrate the spread of direct flux (sunlight) for a space with clear glass using various resolutions of BSDF and sky divisions for the mkillum and rtcontrib tools. The rpict rendering represents "ground truth" or the reference condition against which the percentage difference in 
luminance levels are indicated in Figure $13 .{ }^{4}$ Note how the differences are greatest for both the view portion of the window wall and interior space for the low-resolution cases and decreases as resolution is increased. Resolution of small-area glare sources and the luminance patterns of the windows are also affected, skewing assessments of visual discomfort. The apparent window luminance is averaged over outgoing Klems patches, effectively replacing small intense sources like the sun orb with a larger, less intense glare source (Figure 14).

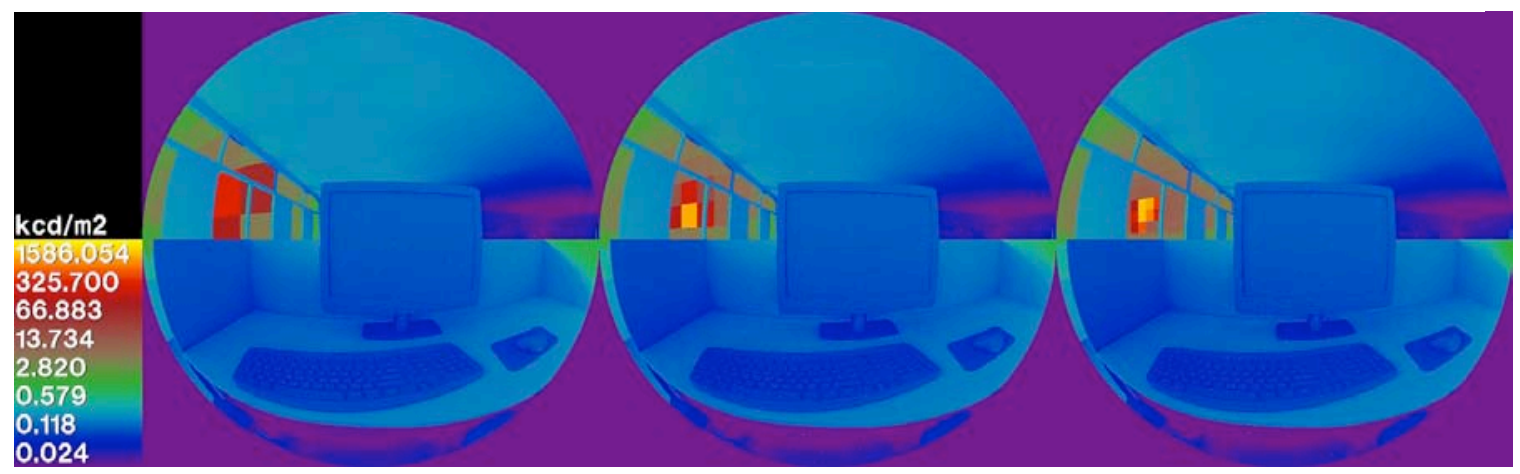

Fig. 14. Rendered views of the sun orb through a window in an office space with clear glazing. From left to right the renderings use full, 2x, and 4x Klems BSDF angle basis. All are rendered with Reinhart MF:4 sky division scheme.

In some cases, an averaged result may be more desirable than a discontinuous pattern. For example, when evaluating work plane illuminance, the real world result depends on the position of the simulation point within the shadow pattern. Whether a high sunlight illuminance or a low shadow illuminance is reported is left to chance. The result from an averaged continuous BSDF output more closely resembles the average illuminance on the work plane. An averaged result may also be more desirable for a viewpoint-specific glare assessment. In the non-BSDF case, the uncertainty of reporting either a higher degree of discomfort or no discomfort is based on the chance of the viewpoint being in sun or shadow. The average BSDF result will report discomfort less than the sun condition but more than the shadow condition. Clearly, simulations using higher-resolution datasets will yield greater accuracy, particularly for peaky systems, but impacts on annual energy and comfort assessments will need to be further investigated.

\subsection{Subdivision of window groups and window}

Use of rtcontrib requires intelligent partitioning of windows into groups to account for nearby obstructions such as an overhang, operable shades whose height varies on a time-step basis, and interactions both inside and outside a building. Inaccuracies can result if this subdivision is not well considered.

The $\mathbf{D}$ matrix contains DC contributions either for a single point on the facade or integrated over an area of the facade. To obtain a more accurate value of incident flux on the window, a simulation may need to be split into many parts with more than one $\mathbf{D}$ matrix to reproduce non-uniform shading effects caused by external obstructions. The number of $\mathbf{D}$ matrices required depends on the proximity and size of external obstructions (Figures 15-17). Similarly, an attached building obstruction may require subdivision of individual windows. Subdividing a window with an overhang into horizontal bands, for example, reproduces the variation in shading over the height of the window provided by the overhang (Figures 1819).

Window groupings and subdivisions used for the $\mathbf{V}$ matrix should mirror those used for the $\mathbf{D}$ matrix. For some cases (i.e., variable height shading systems), it is desirable to divide the windows further for the $\mathbf{V}$ matrix computation. Further division for $\mathbf{V}$ matrix computation is permissible as long as $\mathbf{D}$ matrix groups are reflected in $\mathbf{V}$ matrix subgroups. For example, variable-height shading systems, such as a roller shade,

\footnotetext{
${ }^{4}$ These examples use clear glass which can be defined natively by Radiance and many other simulation software packages. Using a BSDF to simulate clear glass is unnecessary, however is done here to illustrate errors associated with a peaky BSDF that can be understood intuitively.
} 
require at least two BSDFs to represent optical properties above and below the bottom of the shade Figure 20 shows a window divided into 4 equal bands enabling simulation of discrete shade height positions in $25 \%$ increments.

Currently, Radiance does not perform automatic grouping or subdivision of windows. The user is left to determine when window grouping and subdivision is necessary and to determine the appropriate level of subdivision for their simulation needs.
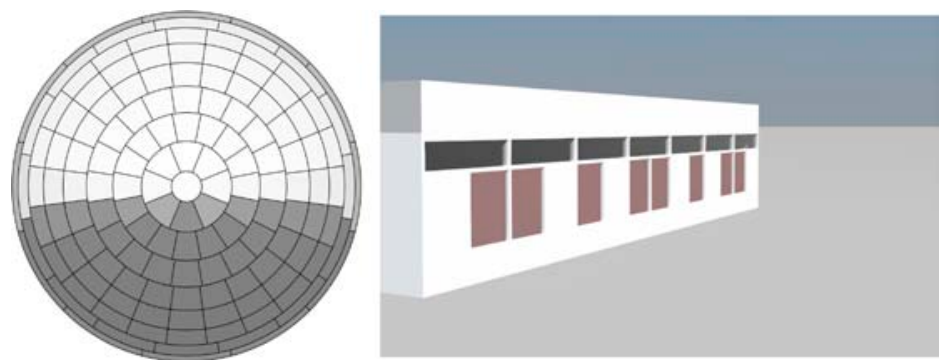

Fig. 15. A visualization of an incident daylight matrix (cumulative coefficients) for all of the view windows (shaded pink).
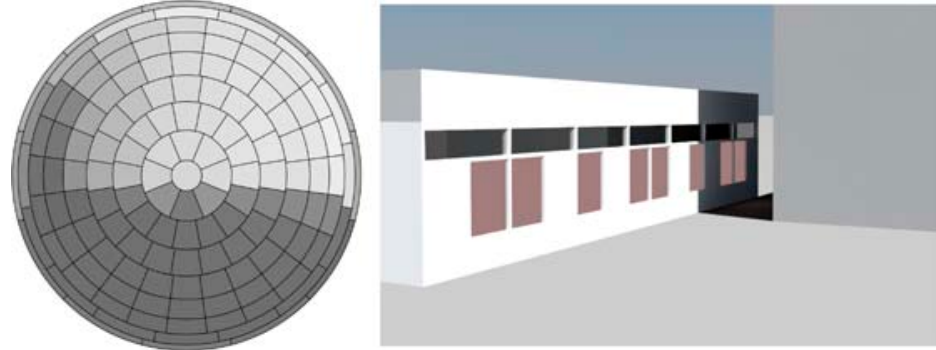

Fig. 16. A visualization of an incident daylight matrix (cumulative coefficients) for all of view windows (shaded pink).

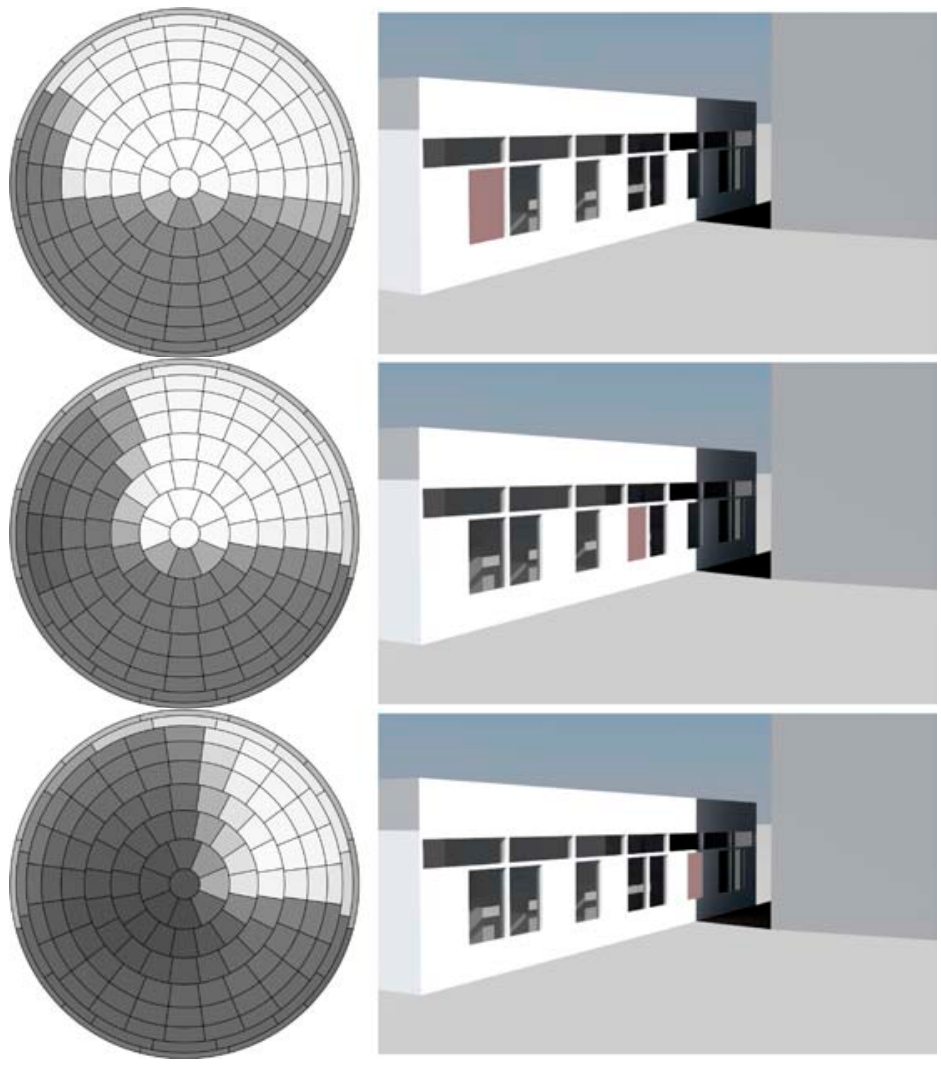




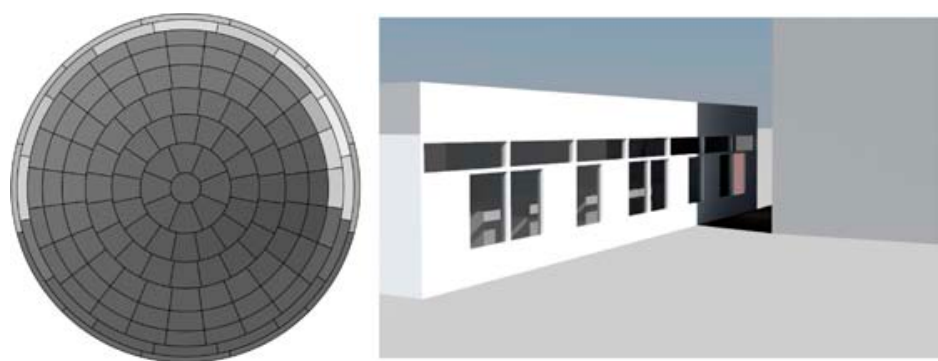

Fig. 17. Visualizations of an incident daylight matrix (cumulative coefficients) for individual windows (shaded pink).

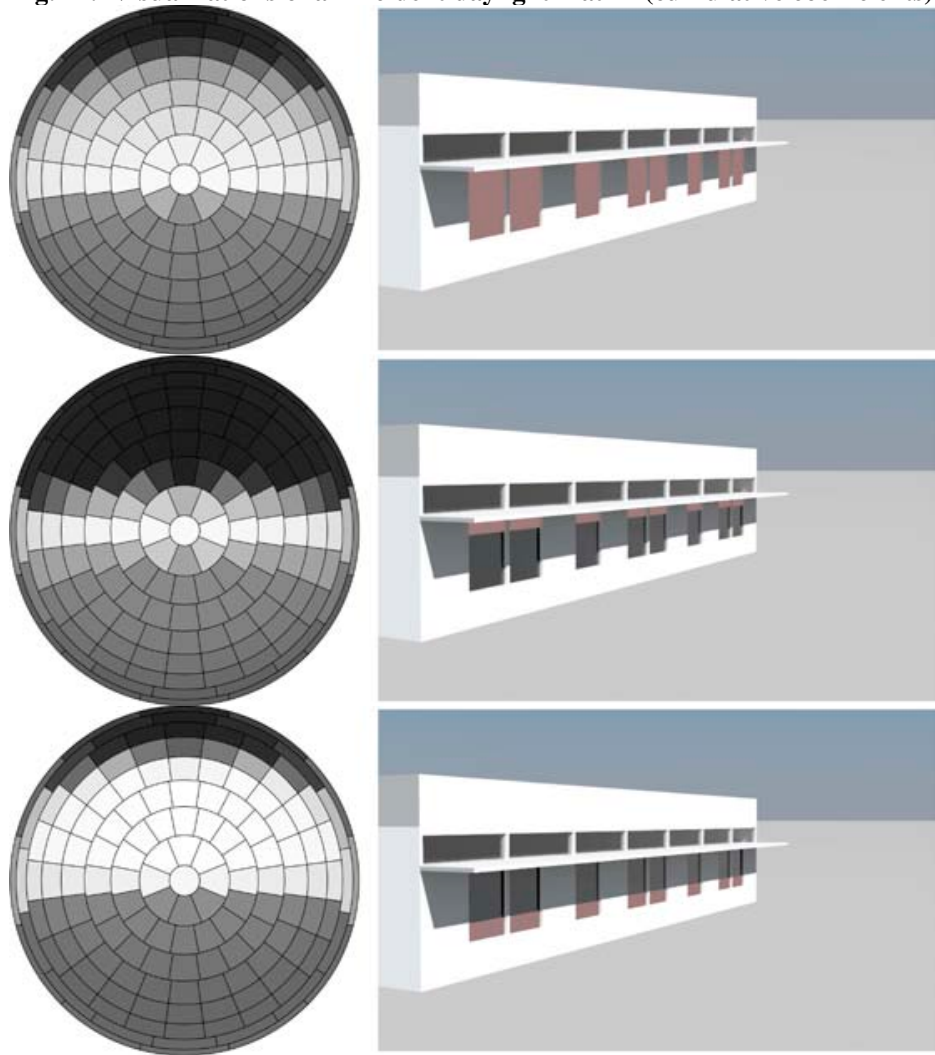

Fig. 18. a) top: Visualization of an incident daylight matrix (cumulative coefficients) for all of view windows (shaded pink, top), then b-c) visualizations of an incident daylight matrix (cumulative coefficients) for the highest and lowest one-quarter subdivisions of the view window.

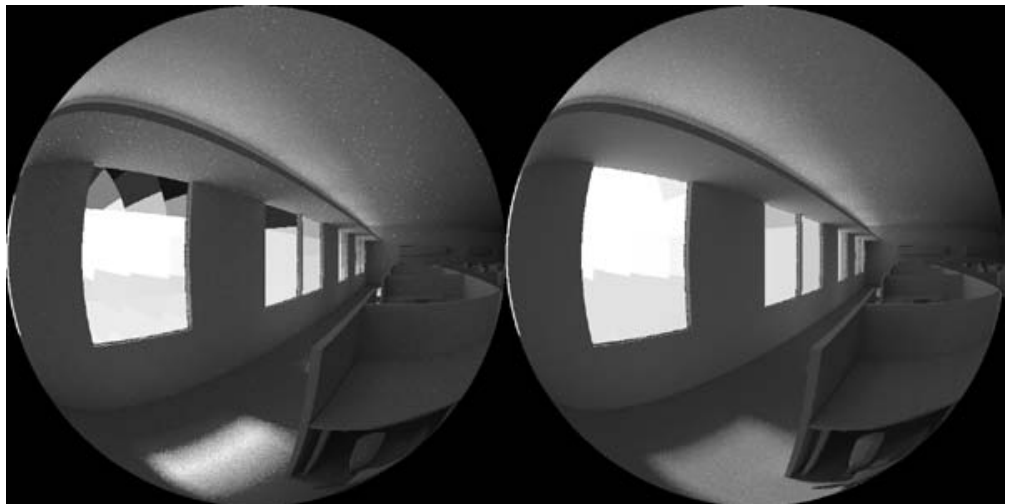

Fig. 19. Interior rtcontrib renderings of a window with an external overhang. When computing the $D$ Matrix the windows are horizontally subdivided (as in Figure 18a in the left image and not subdivided in the right image (as in Figure 18b-c). 


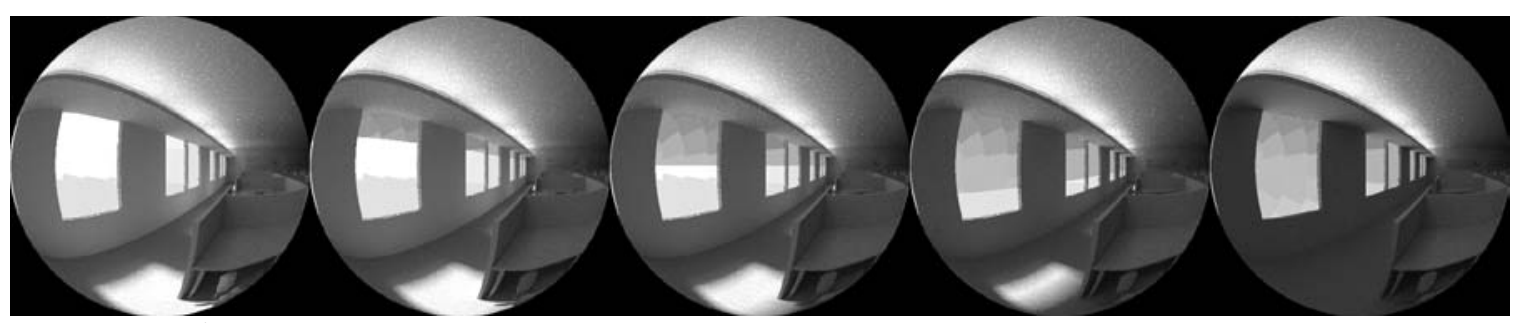

Fig. 20. rtcontrib renderings with venetian blinds covering $0 \%, 25 \%, 50 \%, 75 \%$ and $100 \%$ of the windows.

\section{Conclusions}

A new method of modeling the daylighting performance of complex fenestration systems (CFS) was implemented in Radiance. The method relies on bidirectional scattering distribution functions data output from Window 6, which enables end users to describe any arbitrary assembly of glazing, shading, and other optically-complex coplanar layers. Modifications to two of Radiance's existing tools, mkillum and rtcontrib, enable end users to accurately render an image of an interior space, including shadows cast by an inhomogenous system such as a Venetian blind, and perform efficient annual simulations. The modified tools will require judicious use in order to obtain accurate results. Further work is required to determine the sensitivity of annual and time-step evaluations of peaky systems when resolution of the BSDF basis and sky subdivisions is varied.

\section{Acknowledgments}

We gratefully acknowledge the contributions of Marilyne Andersen, École Polytechnique Federale de Lausanne, toward the validation of this new capability. This work was supported by the Assistant Secretary for Energy Efficiency and Renewable Energy, Building Technologies Program, of the U.S. Department of Energy under Contract No. DE-AC02-05CH11231. This research used the Lawrencium computational cluster resource provided by the IT Division at the Lawrence Berkeley National Laboratory (Supported by the Director, Office of Science, Office of Basic Energy Sciences, of the U.S. Department of Energy under Contract No. DE-AC02-05CH11231).

\section{References}

Andersen M, Stokes E, Gayeski N, Browne C. 2009. Using digital imaging to assess spectral solar-optical properties of complex fenestration materials: A new approach in video-goniophotometry. Sol Energy (2009), doi: 10.1016/j.solener.2009.02.005.

Andersen M, de Boer J. 2006. Goniophotometry and assessment of bi-directional photometric properties of complex fenestration systems, Energy and Buildings 38: 836-848.

Carroll WL, Hitchcock RJ. 2005. DElight2 daylighting analysis in EnergyPlus: Integration and preliminary user results. Proceedings of Building Simulation 2005, Montreal, Canada.

de Boer J. 2006. Modelling indoor illumination by complex fenestration systems based on bidirectional photometric data. Energy and Buildings 38 (2006) 849-868.

Jonsson JC, Rubin M, Nilsson AM, Jonsson A, Roos A. 2009. Optical characteriation of fritted glass for architectural applications. Optical Materials (31): 949-958.

Kaempf JH. 2004. Integration of BT(R)DF data into Radiance lighting simulation programme. Technical report CTI Project 4881.1 Bidrectional Goniophotometer. Ecole Polytechnique Federale de Lausanne.

Klems JH. 1994a. A new method for predicting the solar heat gain of complex fenestration systems: I. Overview and derivation of the matrix layer calculation. ASHRAE Transactions 100 (1): 1065-1072.

Klems JH. 1994b. A new method for predicting the solar heat gain of complex fenestration systems: II. Detailed description of the matrix layer calculation. ASHRAE Transactions 100 (1): 1073-1086. 
Klems JH, Warner JL, Kelley GO. 1997. A comparison between calculated and measured SHGC for complex glazing systems. ASHRAE Transactions (102) 1: 931-939.

Mitchell R, Kohler C, Klems J, Rubin M, Arasteh D, Huizenga C, Yu T, Curcijaf D, editors. 2008. Window 6.2/ Therm 6.2 Research Version User Manual. Lawrence Berkeley National Laboratory. LBNL-941.

Berkeley, CA 94720.

Nicodemus FE. 1965. Directional Reflectance and Emissivity of an Opaque Surface. Appl Opt 4. 767-773.

Reinhart CF. 2001. Daylight availability and manual lighting control in office buildings - Simulation studies and analysis of measurements. Thesis. University of Karlsruhe.

Saxena M, Ward G, Perry T, Heschong L, Higa R. 2010. Dynamic Radiance - Predicting annual daylighting with variable fenestration optics using BSDFs. Proceedings of SimBuild 2010. 4th National Conference of IBPSA-USA. 2010 August 11-13. New York.

Schregle R, Weinold J. 2004. Physical Validation of Global Illumination Methods: Measurement and Error Analysis. Computer Graphics Forum. Vol 23. No 4. 761-81.

Tregenza PR. 1983. Daylight coefficients. Lighting Research and Technology. Vol 15. No 2. 65-71.

Larson, Ward G, Shakespeare R. 1998. Rendering with Radiance: The Art and Science of Lighting Visualization. San Francisco. Morgan Kaufmann. 